\title{
Environmental Effects on Nonlocal Correlations
}

\author{
Tamal Guha ${ }^{1}$, Bihalan Bhattacharya ${ }^{2}$, Debarshi Das ${ }^{3}$, Some Sankar Bhattacharya ${ }^{4}$, \\ Amit Mukherjee ${ }^{2,5}$, Arup Roy ${ }^{2}$, Kaushiki Mukherjee ${ }^{6}$, Nirman Ganguly ${ }^{7}$ \\ \& A. S. Majumdar ${ }^{2 \dagger}$
}

${ }^{1}$ Physics and Applied Mathematics Unit, Indian Statistical Institute, Kolkata, India

${ }^{2}$ S. N. Bose National Centre for Basic Sciences, Salt Lake, Kolkata, India

${ }^{3}$ Centre for Astroparticle Physics and Space Science, Bose Institute, Salt Lake, Kolkata, India

${ }^{4}$ Department of Computer Science, University of Hong Kong, Hong Kong

${ }^{5}$ Optics and Quantum Information Group, Institute of Mathematical Sciences, HBNI, C.I.T. Campus, Taramani, Chennai, India

${ }^{6}$ Department of Mathematics, Government Girls General Degree College, Ekbalpore, Kolkata, India

7 Department of Mathematics, Birla Institute of Technology and Science Pilani, Hyderabad Campus, Telengana, India

† Corresponding author. E-mail:archan@bose.res.in

Editors: Srikanth Radhakrishna \& Danko Georgiev

Article history: Submitted on April 17, 2019; Accepted on October 19, 2019; Published on October 28, 2019.

E nvironmental interactions are ubiquitous in practical instances of any quantum information processing protocol. The interaction results in depletion of various quantum resources and even complete loss in numerous situations. Nonlocality, which is one particular quantum resource marking a significant departure of quantum mechanics from classical mechanics, meets the same fate. In the present work we study the decay in nonlocality to the extent of the output state admitting a local hidden state model. Using some fundamental quantum channels we also demonstrate the complete decay in the resources in the purview of the Bell-ClauserHorne-Shimony-Holt inequality and a three-settings steering inequality. We also obtain bounds on the parameter of the depolarizing map for which it becomes steerability breaking pertaining to a general class of two qubit states.

Quanta 2019; 8: 57-67.

(c) (i) This is an open access article distributed under the terms of the Creative Commons Attribution License CC-BY-3.0, which permits unrestricted use, distribution, and reproduction in any medium, provided the original author and source are credited.

\section{Introduction}

Nonlocality [1] is one of the key features that sets quantum mechanics apart from classical mechanics. Quantum nonlocality is generally interpreted as the failure to describe quantum mechanical correlations arising due to spacelike separated local quantum measurements on subsystems of a composite system by local realist models. Bell inequalities [2] are used to reveal such incompatibility between quantum mechanics and local-realism. A state which satisfies a set of Bell inequalities cannot be guaranteed as local, as there may exist another set of Bell inequalities that it violates. On the other hand, quantum mechanical violation of any Bell-Clauser-HorneShimony-Holt (Bell-CHSH) inequality is a signature of quantum nonlocality [3]. However, the complete set of Bell-CHSH inequalities is the necessary and sufficient criterion for local-realism in the 2-2-2 experimental scenario ( 2 parties, 2 measurement settings per party, 2 outcomes per measurement setting). A state is termed as local only if the correlations arising by performing local quantum measurements on it admit a local hidden variable (LHV) model [4].

The pioneering study by Einstein, Podolsky and Rosen (EPR) [5] arguing for incompleteness of the quantum 
mechanical description of reality motivated Schrödinger to propose the concept of quantum steering [6,7]. EPR steering arises in the scenario where local quantum measurements on one part of a bipartite spatially separated system allow to prepare different ensembles on the other part. This scenario demonstrates EPR steering if these ensembles cannot be explained by a local hidden state (LHS) model [8,9]. This kind of interpretation of steering has induced great interest in foundational research in recent times as evidenced by a wide range of studies [10-20]. Reid first proposed a criterion for testing EPR-steering in continuous-variable systems based on position-momentum uncertainty relation [21], which was experimentally tested by Ou et al. [22]. More recently, Cavalcanti et al. constructed experimental EPR-steering criteria based on the assumption of the existence of LHS model [23]. This general construction is applicable to discrete as well as continuous-variable observables and Reid's criterion appears as a special case of this general formulation.

More importantly, entanglement [24] is a necessary condition for the demonstration of Bell-nonlocality or steerability. The inequivalence of entanglement and nonlocality is exemplified by showing the existence of certain entangled states producing quantum mechanical correlations that admit a LHV model [25]. Bell-nonlocal states form a strict subset of steerable states which also form a strict subset of entangled states [8,26].

Apart from being important candidates in foundational studies of quantum mechanics, quantum nonlocal, steerable and entangled states serve as resource in various quantum information processing tasks, for example, teleportation [27], randomness certification [28-30], cryptography [31, 32] and so on. Motivated by this fact a number of studies have been performed towards revealing hidden nonlocality from quantum states that failed to demonstrate nonlocality under the standard Bell scenario [1]. Local filtering operation is one such procedure [33, 34], which can be broadly classified into two categories: (i) performing single local measurement [34] and (ii) subjecting the state to suitable sequence of local measurements [33]. Similarly, the issue of revealing hidden quantum steerability by using local filters has also been studied [26, 35].

In practical scenarios, a state is subjected to ubiquitous environmental interaction and hence may lose its entanglement or nonlocal character partially or completely. Thus, it is of considerable interest to study the behaviour of entangled states as well as nonlocal states under local noise. The issue related to entanglement breaking channels which transform an entangled state into a separable one, has acquired a lot of significance as witnessed by a number of studies [36, 37]. On the other hand, studies on nonlocality breaking maps, which transform a
Bell-CHSH nonlocal state into a local one, have also probed the role of environment in destroying quantum resources [38]. Recently, a general framework for analyzing resource theories based on resource destroying maps has been proposed [39]. In this context, one may also consider the case of incompatibility breaking maps [40], as incompatibility of quantum measurements is an important resource in quantum information. The study of such maps is intriguing as steerability and incompatibility have a one to one correspondence [41].

A separable state can be transformed into an entangled one when subjected to a global unitary action acting on the composite system. However, it has been shown that there are separable states, dubbed as absolutely separable states, which cannot be transformed into an entangled one under any global unitary interaction [42-45]. Recently, the effect of global unitary interactions on the nonlocality of a state has been probed, with the focus on the Bell$\mathrm{CHSH}$ inequality for two qubit systems. A state initially satisfying the Bell-CHSH inequality can violate it after a global unitary interaction. On the other hand, it has been demonstrated that there are states which preserve their Bell-CHSH local character under arbitrary global unitary action. These states are termed as absolutely BellCHSH local states [46, 47]. The question of transforming a separable, but not absolutely separable, state into an absolutely separable one under environmental interactions is important in practical situations and has been investigated recently [48].

In the context of absolutely Bell-CHSH local states, the issue of transforming an absolutely Bell-CHSH local state into a nonlocal one has also been presented in a recent study [49]. In a similar spirit, the effect of global unitary interactions on states demonstrating EPR steering has also been studied [50] in the context of steering inequalities derived by Cavalcanti et al. [23]. In particular, the issue of non-violation of the steering inequality with three measurement settings per party [23] by any two qubit system under arbitrary global unitary action has been studied in details. For our convenience, we will denote the states which preserve their non-violation of the steering inequality with three measurement settings per party derived by Cavalcanti et al. [23] under arbitrary global unitary action as absolutely three-settings unsteerable states. Studies have shown that any pure state cannot be absolutely BellCHSH local, or absolutely three-settings unsteerable, or absolutely separable. However, one must remember that environmental interactions bound us to work with mixed states where such phenomena are practically very possible. Therefore, one can also understand the significance of such confrontations even through the lens of quantum information processing protocols. 
A natural question arising in this context is what are the possible instances that one should avoid while working with nonlocal resources under the influence of environment. In the present work we provide such illustrations with commonly used quantum channels. Our study is done in the purview of Bell-CHSH inequality and the three-settings steering inequality mentioned in the text. The resultant states do not violate the inequalities even after consuming the power of global unitary operations. This is where the present study departs from previous works as it probes the feature that nonlocality may be non-retrievable even with global unitary operations.

On the foundational significance of our work, we have been able to generate entangled states which admit LHS models from initially nonlocal state. Werner, in his seminal paper [25] had proved the existence of entangled states having LHV. That is, entangled states exist which cannot give rise to nonlocality under arbitrary (non-sequential) measurements. There is a recent spurt in research in constructing entangled states having LHV or LHS model [51.52] using semi-definite programming. To put matters in context, let Alice and Bob share a quantum state. Alice performs a measurement $x \in\left\{x_{0}, x_{1}, x_{2}, \ldots, x_{n}\right\}$ and obtains an outcome $a \in\left\{0,1,2, \ldots, d_{A}\right\}$ and Bob performs a measurement $y \in\left\{y_{0}, y_{1}, y_{2}, \ldots, y_{m}\right\}$ and obtains an outcome $b$ $\in\left\{0,1,2, \ldots, d_{B}\right\}$. If they repeat these trials many times, they generate a correlation $p(a b \mid x y)$. The joint correlation is said to have LHV model if and only if $p(a b \mid x y)=$ $\sum_{\lambda} \rho(\lambda) p(a \mid x, \lambda) p(b \mid y, \lambda)$, where $p(a \mid x, \lambda)$ and $p(b \mid y, \lambda)$ are arbitrary distributions conditioned on LHV $\lambda$. On the other hand, the joint correlation is said to have LHS model if and only if $p(a b \mid x y)=\sum_{\lambda} \rho(\lambda) p(a \mid x, \lambda) p\left(b \mid y, \sigma_{\lambda}\right)$, where $p(a \mid x, \lambda)$ is arbitrary distribution conditioned on LHV $\lambda, p\left(b \mid y, \sigma_{\lambda}\right)$ is the quantum probability of obtaining the outcome $b$ when the measurement $y$ is performed on the local hidden state $\sigma_{\lambda}$ (which is a quantum state). In our present work we have been able to generate an entangled state admitting LHS model due to environmental influence.

Pure entangled states always violate a suitably chosen Bell's inequality. However, purity is fragile under environmental influences and thus, mixed states are more common in experimental scenarios. Mixed entangled states can have a LHV model, the most celebrated example being of the Werner state in two qubits. Therefore, our present work deals with mixed entangled states.

We have studied the context of steerability breaking maps. Particularly we have obtained bounds on the parameter of the depolarizing map such that it becomes steerability breaking pertaining to a general class of two qubit states. This is particularly significant because steerability is a weaker form of nonlocality as compared to Bell nonlocality.
The paper has been arranged in the following way. Starting from some preliminary discussions in Section 2 we have illustrated the scenarios in Section 3 In Section 4 , we have studied the transformation of Bell-CHSH nonlocal state to Bell-CHSH local, absolutely Bell-CHSH local and absolutely three-settings unsteerable states under environmental interactions, followed by one of the highlights of our analysis, viz., generation of entangled states admitting LHS model from initially nonlocal state using two different quantum channels in Section 5 . In Section 6, we discuss on the analysis of steerability breaking channels in the context of the depolarizing map which is another interesting attribute of our approach. Finally, we conclude along with a discussion in Section 7.

\section{Preliminaries}

Let us start with some preliminary ideas required for the present study.

\subsection{Bell-CHSH locality}

A bipartite state is said to be Bell-CHSH local if and only if (iff) the correlations obtained by performing local quantum measurements on the two subsystems of the composite state (where the local measurements performed on one subsystem are spacelike separated from that on another subsystem) do not violate the Bell-CHSH inequality. The necessary and sufficient criteria for quantum mechanical violation of the CHSH inequality by arbitrary bipartite qubit states has been established in [53].

An arbitrary two qubit state can be expressed in terms of the Hilbert-Schmidt basis as

$$
\rho=\frac{1}{4}\left(\mathbb{I} \otimes \mathbb{I}+\vec{r} . \vec{\sigma} \otimes \mathbb{I}+\mathbb{I} \otimes \vec{s} . \vec{\sigma}+\sum_{i, j=1}^{3} t_{i j} \sigma_{i} \otimes \sigma_{j}\right) .
$$

Here $\mathbb{I}$ is the identity operator acting on $\mathbb{C}^{2} ; \sigma_{i}$ s are the three Pauli matrices; $\vec{r}, \vec{s}$ are vectors in $\mathbb{R}^{3}$ with norm less than or equal to unity; $\vec{r} . \vec{\sigma}=\sum_{i=1}^{3} r_{i} \sigma_{i}$ and $\vec{s} . \vec{\sigma}=$ $\sum_{i=1}^{3} s_{i} \sigma_{i}$. The condition $\operatorname{Tr}\left(\rho^{2}\right) \leq 1$ implies

$$
\sum_{i=1}^{3}\left(r_{i}^{2}+s_{i}^{2}\right)+\sum_{i, j=1}^{3} t_{i j}^{2} \leq 3
$$

where the equality is achieved for the pure states. In addition, for being a valid density matrix, $\rho$ has to be positive semidefinite.

Let us consider the matrix $V=T^{\dagger} T$, where $T$ is the correlation matrix of the state (1) with matrix elements $t_{i j}=\operatorname{Tr}\left(\rho \sigma_{i} \otimes \sigma_{j}\right)$. Denote with $u_{1}, u_{2}$ the two greatest eigenvalues of $V$. Let us consider the quantity given by

$$
M(\rho)=u_{1}+u_{2} .
$$


The state given by Eq. (1) violates the Bell-CHSH inequality iff $M(\rho)>1$. Hence, the state (1) is Bell-CHSH local iff $M(\rho) \leq 1$.

\subsection{Absolute Bell-CHSH locality}

The concept of absolutely Bell-CHSH local states has recently been introduced in [46]. A Bell-CHSH local quantum state is said to be absolutely Bell-CHSH local if the state remains Bell-CHSH local under the action of any global unitary operation. If $a_{1}, a_{2}, a_{3}$ are the three largest eigenvalues of the given two qubit state $\rho$ taken in descending order, then the state $\rho$ is absolutely BellCHSH local iff [47]

$$
A(\rho)=\left(2 a_{1}+2 a_{2}-1\right)^{2}+\left(2 a_{1}+2 a_{3}-1\right)^{2} \leq 1 .
$$

\subsection{Absolute three-settings unsteerability}

Cavalcanti et al. have provided a series of steering inequalities to certify whether a bipartite state is steerable when each of the two parties are allowed to perform $n$ measurements on his or her part [23]. In particular for $n=3$, the inequality is given by,

$$
F^{3}=\frac{1}{\sqrt{3}}\left|\sum_{i=1}^{3}\left\langle A_{i} \otimes B_{i}\right\rangle\right| \leq 1,
$$

where, $A_{i}=\hat{u_{i}} \cdot \vec{\sigma}, B_{i}=\hat{v_{i}} \cdot \vec{\sigma}, \vec{\sigma}=\left(\sigma_{1}, \sigma_{2}, \sigma_{3}\right)$ is a vector composed of Pauli matrices, $\hat{u}_{i} \in \mathbb{R}^{3}$ are unit vectors, $\hat{v}_{i} \in \mathbb{R}^{3}$ are orthonormal vectors. $\left\langle A_{i} \otimes B_{i}\right\rangle=\operatorname{Tr}\left(\rho A_{i} \otimes B_{i}\right)$ with $\rho \in \mathcal{B}\left(\mathcal{H}_{A} \otimes \mathcal{H}_{B}\right)$ is the bipartite quantum system shared between the two parties.

The states, which preserve their non-violation of the steering inequality (5) under arbitrary global unitary action, are called absolutely three-settings unsteerable states. A given two qubit state $\rho$ is absolutely threesettings unsteerable iff [50]

$$
\begin{aligned}
B(\rho)=3 \operatorname{Tr}\left(\rho^{2}\right)-2 & \left(x_{1} x_{2}+x_{1} x_{3}+x_{1} x_{4}\right. \\
& \left.+x_{2} x_{3}+x_{2} x_{4}+x_{3} x_{4}\right) \leq 1,
\end{aligned}
$$

where $x_{i}$ are the eigenvalues of the two qubit state $\rho$.

\subsection{Quantum channels}

In practical scenarios it is very hard to isolate a qubit from its environment. Environmental interactions can be represented by different quantum channels. Quantum channels are completely positive trace preserving (CPTP) maps acting on the space of density matrices [54]. Every quantum channel admits the operator sum representation.
Let $\varepsilon$ be a quantum channel, then its action on the state $\tilde{\rho}$ can be expressed as

$$
\varepsilon(\tilde{\rho})=\sum_{i} K_{i} \tilde{\rho} K_{i}^{\dagger},
$$

where $K_{i}$ 's are Kraus operators for the corresponding channel with $\sum_{i} K_{i}^{\dagger} K_{i}=\mathbb{I}$ ( $\mathbb{I}$ is the identity operator). In the present study we restrict ourselves to four quantum channels, viz. 1) phase-flip channel ,2) bit-flip channel, 3) depolarizing channel, and 4) phase damping channel.

\subsubsection{Phase-flip channel}

The action of the phase-flip channel on the state $\tilde{\rho}$ is given by Eq. (7) with the following Kraus operators [54]

$$
\begin{aligned}
& K_{0}=\sqrt{1-p} \mathbb{I}, \\
& K_{1}=\sqrt{p} \sigma_{z},
\end{aligned}
$$

where $p$ is the channel strength with $0 \leq p \leq 1$.

\subsubsection{Bit-flip channel}

The action of the bit-flip channel on the state $\tilde{\rho}$ is given by Eq. (7) with the following Kraus operators [54]

$$
\begin{aligned}
& K_{0}=\sqrt{1-p} \mathbb{I}, \\
& K_{1}=\sqrt{p} \sigma_{x}
\end{aligned}
$$

where $p$ is the channel strength with $0 \leq p \leq 1$.

\subsubsection{Depolarizing channel}

The action of the depolarizing channel on the state $\tilde{\rho}$ is given by Eq. (7) with the following Kraus operators [54]

$$
\begin{aligned}
& K_{0}=\sqrt{1-p} \mathbb{I}, \\
& K_{1}=\sqrt{\frac{p}{3}} \sigma_{x}, \\
& K_{2}=\sqrt{\frac{p}{3}} \sigma_{y}, \\
& K_{3}=\sqrt{\frac{p}{3}} \sigma_{z},
\end{aligned}
$$

where $p$ is the channel strength with $0 \leq p \leq 1$.

\subsubsection{Phase damping channel}

The action of the phase damping channel on the state $\tilde{\rho}$ is given by Eq. (7) with the following Kraus operators [54]

$$
\begin{aligned}
K_{0} & =\left[\begin{array}{cc}
1 & 0 \\
0 & \sqrt{1-p}
\end{array}\right], \\
K_{1} & =\left[\begin{array}{cc}
0 & 0 \\
0 & \sqrt{p}
\end{array}\right]
\end{aligned}
$$

Here $p$ is the channel strength with $0 \leq p \leq 1$. 


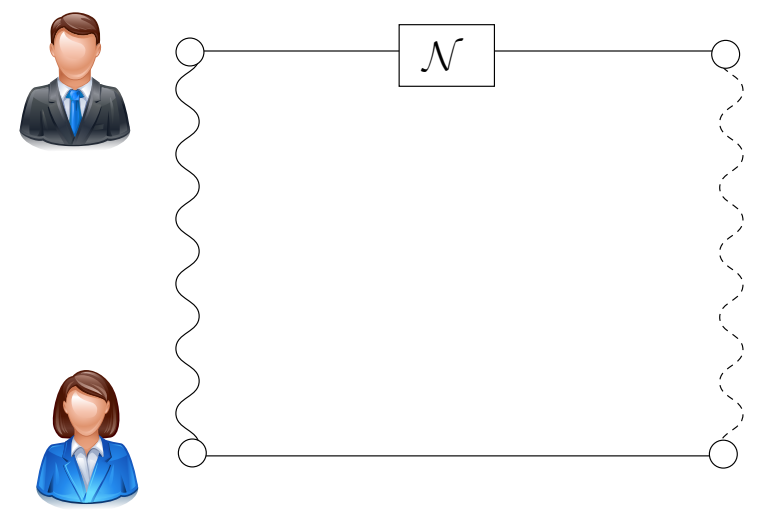

Figure 1: Single interaction scenario where $\mathcal{N}$ stands for local channel acting on Bob's side.

\section{The Scenarios}

In order to study the effect of environmental interactions on nonlocal states, we have considered two scenarios as follows.

\subsection{Single interaction}

Consider that Alice and Bob (the set of local measurements performed by Alice is spacelike separated from that of Bob) share a nonlocal two qubit mixed state. Bob's qubit interacts with the environment simulated by a quantum channel of strength $p$. They finally obtain a family of mixed states which are dependent on $p$. We calculate the range of the channel strengths for which the state becomes Bell-CHSH local, absolutely Bell-CHSH local, absolutely three-settings unsteerable. Figure 1 depicts the scenario.

\subsection{Double Interaction}

In this case the situation differs from the above in the sense that now both the qubits interact with environment. The quantum channel is of the same strength $p$. In this case too we obtain the spread of the channel strength pertaining to the same manifestations as above. The scenario is represented by Figure 2

Next, we present the first significant result of our work.
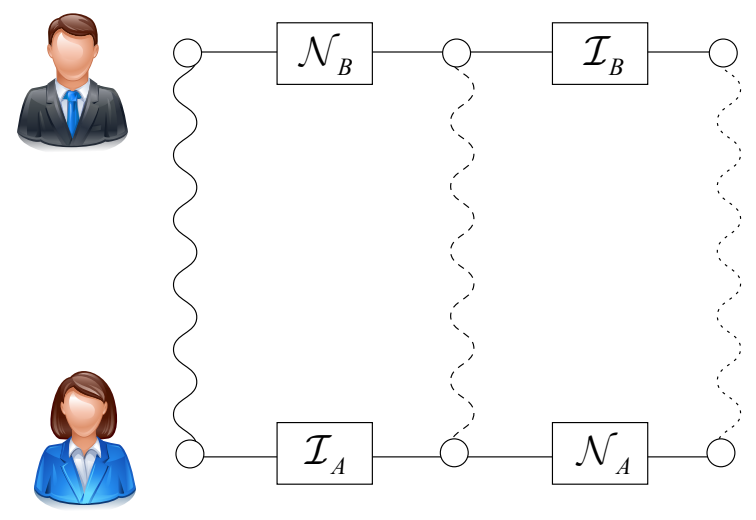

Figure 2: Sequential interaction where $\mathcal{N}_{B}$ and $\mathcal{N}_{A}$ stand for local channels acting on Bob's and Alice's side respectively. $\mathcal{I}_{B}$ and $\mathcal{I}_{A}$ represent identity operations on respective sides.

\section{From nonlocal state to Bell-CHSH local state, absolutely Bell-CHSH local state and absolutely three-settings unsteerable state}

Let us consider that the following two parameter family of mixed states is shared among Alice and Bob where the local measurements performed by Alice are spacelike separated from that of Bob

$$
\rho_{i}(\lambda, \theta)=\left[\begin{array}{cccc}
\frac{1-\lambda}{2} & 0 & 0 & 0 \\
0 & \lambda \sin ^{2} \theta & \frac{\lambda}{2} \sin 2 \theta & 0 \\
0 & \frac{\lambda}{2} \sin 2 \theta & \lambda \cos ^{2} \theta & 0 \\
0 & 0 & 0 & \frac{1-\lambda}{2}
\end{array}\right] .
$$

Figure 3 depicts the nonlocal region for the family of mixed states given by Eq. (8). Let us choose two initial states from the above two parameter family of states, one with $\lambda=0.95, \theta=0.6$ and another with $\lambda=0.80$ and $\theta=0.6$, such that the two initial states are nonlocal. Let us consider that the initial states are subjected to the aforementioned single and double interactions of different quantum channels with channel strength $p$. Let $R_{1}, R_{2}$ and $R_{3}$ denote the ranges of $p$ for which the states obtained are Bell-CHSH local, absolutely Bell-CHSH local, and absolutely three-settings unsteerable, respectively.

In what follows below we have tabulated the various manifestations under different channel parameters for the single and double interactions respectively.

Tables 1 and 2 show the effect of single and sequential interaction of different channels with respect to two different initial states.

It is significant to note that for bit-flip and phase-flip channels, along with the lower bound there is also an upper bound on the noise parameter $(p)$, between which they 


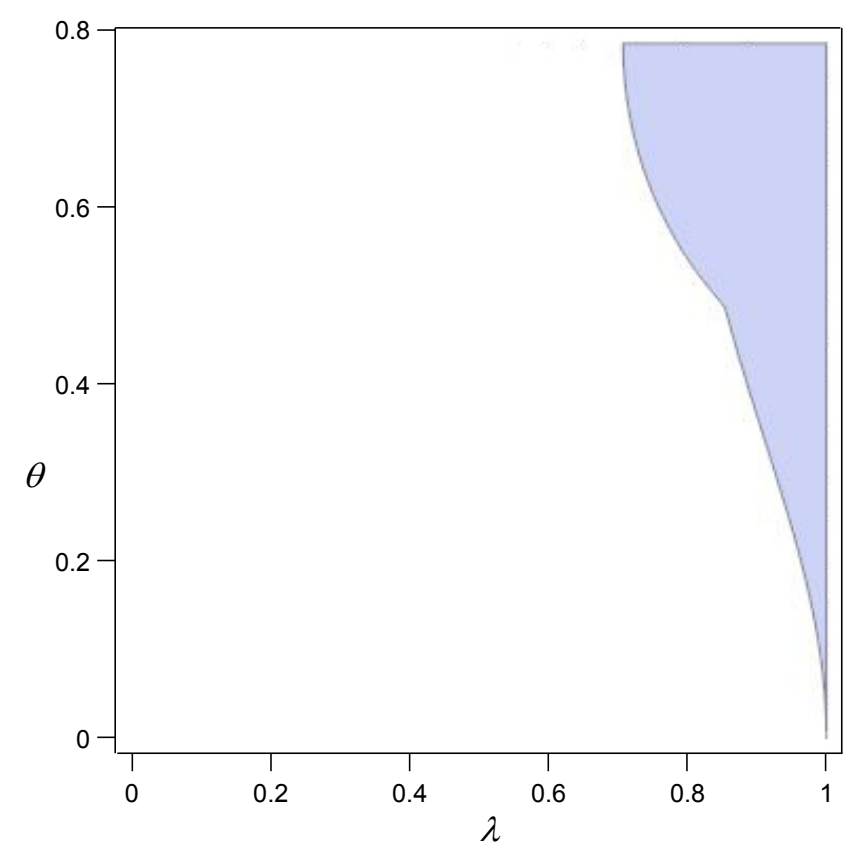

Figure 3: The horizontal axis represents $\lambda$ and the vertical axis represents $\theta$. The shaded region depicts the nonlocal region of the state (8).

act as resource destroying maps. This counter-intuitive feature has a deep connection with the structure of the state (8). Single interaction of the phase flip noise makes $(\lambda, \theta) \rightarrow(\lambda,-\theta)$, whereas the bit flip noise makes a block relabelling of the initial quantum state. As a result, for the value of noise parameter beyond an upper-bound, the resource destroying power of the map is decreased in case of the above mentioned channels. It is also worthwhile to note that barring the first row and one range in the second row the ranges in Table 1 are subsets of their counterpart in Table 2. This agrees with intuition as Table 2 depicts the range pertaining to double interaction.

\section{Generating entangled states admitting LHS model from initially nonlocal state}

There has been considerable work in recent times on the construction of states admitting LHV and LHS models using techniques from semidefinite programming [51. 52]. In what follows below, we lay down two scenarios which generate entangled states admitting LHS model from initially nonlocal state.

\subsection{The scenario using phase damping channel}

Let us consider the following two parameter family of bipartite two-qubit mixed quantum states initially shared between Alice and Bob (the local measurements performed

Table 1: Single interaction

\begin{tabular}{ccccc}
\hline Channels & Initial state parameters & $R_{1}$ & $R_{2}$ & $R_{3}$ \\
\hline Phase flip & $\lambda=0.95, \theta=0.6$ & {$[0.1492,0.8508]$} & {$[0.2252,0.7747]$} & - \\
\hline Phase flip & $\lambda=0.8, \theta=0.6$ & {$[0.0258,0.9742]$} & {$[0.0675,0.9325]$} & {$[0.1743,0.8257]$} \\
\hline Bit flip & $\lambda=0.95, \theta=0.6$ & {$[0.2445,0.7342]$} & {$[0.3532,0.6443]$} & {$[0.3807,0.6193]$} \\
\hline Bit flip & $\lambda=0.8, \theta=0.6$ & {$[0.0531,0.9468]$} & {$[0.1250,0.8750]$} & {$[0.2,0.8]$} \\
\hline Depolarizing & $\lambda=0.95, \theta=0.6$ & {$[0.0685,1]$} & {$[0.1928,1]$} & {$[0.2893,1]$} \\
\hline Depolarizing & $\lambda=0.8, \theta=0.6$ & {$[0.0692,1]$} & {$[0.1928,1]$} & {$[0.2893,1]$} \\
\hline Phase damping & $\lambda=0.95, \theta=0.6$ & {$[0.3723,1]$} & {$[0.7846,1]$} & - \\
\hline Phase damping & $\lambda=0.8, \theta=0.6$ & {$[0.0516,1]$} & {$[0.1350,1]$} & {$[0.3485,1]$} \\
\hline
\end{tabular}

Table 2: Double interaction

\begin{tabular}{ccccc}
\hline Channels & Initial state parameters & $R_{1}$ & $R_{2}$ & $R_{3}$ \\
\hline Phase flip & $\lambda=0.95, \theta=0.6$ & {$[0.1492,0.8508]$} & {$[0.2252,0.7747]$} & - \\
\hline Phase flip & $\lambda=0.8, \theta=0.6$ & {$[0.0131,0.9869]$} & {$[0.3050,0.9650]$} & {$[0.0964,0.9036]$} \\
\hline Bit flip & $\lambda=0.95, \theta=0.6$ & {$[0.1378,0.8622]$} & {$[0.1856,0.8144]$} & {$[0.2256,0.7744]$} \\
\hline Bit flip & $\lambda=0.8, \theta=0.6$ & {$[0.0273,0.9727]$} & {$[0.0654,0.9345]$} & {$[0.1093,0.8907]$} \\
\hline Depolarizing & $\lambda=0.95, \theta=0.6$ & {$[0.0354,1]$} & {$[0.0727,1]$} & {$[0.1560,1]$} \\
\hline Depolarizing & $\lambda=0.8, \theta=0.6$ & {$[0.0196,1]$} & {$[0.0481,1]$} & {$[0.0922,1]$} \\
\hline Phase damping & $\lambda=0.95, \theta=0.6$ & {$[0.2077,1]$} & {$[0.5359,1]$} & - \\
\hline Phase damping & $\lambda=0.8, \theta=0.6$ & {$[0.0261,1]$} & {$[0.0699,1]$} & {$[0.1928,1]$} \\
\hline
\end{tabular}


by Alice are spacelike separated from that of Bob)

$$
\begin{aligned}
\rho_{i}(q, s)= & q\left(s\left|\phi^{+}\right\rangle\left\langle\phi^{+}\right|+(1-s) \frac{1}{4} \mathbb{I}\right) \\
& +(1-q) \frac{1}{2}(|00\rangle\langle 00|+| 11\rangle\langle 11|)
\end{aligned}
$$

where $|0\rangle$ and $|1\rangle$ are the eigenstates of the operator $\sigma_{z}$ with eigenvalues +1 and -1 respectively,

$$
\left|\phi^{+}\right\rangle=\frac{1}{\sqrt{2}}(|00\rangle+|11\rangle),
$$

$\mathbb{I}$ is the identity operator, $0 \leq q \leq 1$ and $0 \leq s \leq 1$.

Now, we use the phase damping channel. Let us choose $q=0.96, s=0.74$ such that the initial state given by Eq. (9) is nonlocal. In case of the phase damping channel with channel strength $p$, if this state undergoes single interaction as described earlier, then for $p=p_{1}=0.65$, the state becomes

$$
\rho_{f}=\frac{1}{2} \sigma+\frac{1}{4}(|00\rangle\langle 00|+| 11\rangle\langle 11|),
$$

where $\sigma$ is the two qubit isotropic state given by

$$
\sigma=\frac{1}{2}\left(\left|\phi^{+}\right\rangle\left\langle\phi^{+}\right|+\frac{1}{4} \mathbb{I}\right)
$$

It has been shown that the correlation produced by the state $\rho_{f}$ given by Eq. (10) has a local hidden variablelocal hidden state (LHV-LHS) model [55]. Hence, the correlation produced by the state $\rho_{f}$ has a LHV model, since the states having a LHV-LHS model form a subset of the states having a LHV model. It can easily be checked that the state $\rho_{f}$ is absolutely Bell-CHSH local according to the condition given by (4). The state $\rho_{f}$ is absolutely three-settings unsteerable also according to the condition given by (6).

Again, in case of the phase damping channel with channel strength $p$, if the state $\rho_{i}(q, s)$ given by Eq. (9) (with $q=0.96, s=0.74)$ undergoes sequential interaction as described earlier, then for $p=p_{2}=0.41$ the state becomes $\rho_{f}$ given by Eq. (10).

Hence, here we have presented the transformation of a nonlocal state into an absolutely Bell-CHSH local state (as well as absolutely three-settings unsteerable state) with an LHV model as well as LHS model under single and double interaction of the phase damping channel. It is clear that $p_{2}<p_{1}$, which implies that the state $\rho_{i}(q, s)$ (with $\left.q=0.96, s=0.74\right)$ can be transformed into an absolutely Bell-CHSH local state (as well as an absolutely three-settings unsteerable state) having a LHV model as well as LHS model under double interaction of the phase damping channel with smaller channel strength compared to that under single interaction.

\subsection{The scenario using depolarizing channel}

In this case let us choose $q=0.34, s=0.97$ such that the initial state given by Eq. (9) is nonlocal. In case of the depolarizing channel with channel strength $p$, if this state undergoes single interaction as described earlier, then for $p=p_{1}=0.18$ the state becomes $\rho_{f}$ given by Eq. (10). If the state $\rho_{i}(q, s)$ given by Eq. (9) (with $q=0.34, s=$ 0.97 ) undergoes sequential interaction of the depolarizing channel as described earlier, then for $p=p_{2}=0.10$ the state becomes $\rho_{f}$ given by Eq. (10).

Hence, here we have presented the transformation of a nonlocal state into an absolutely Bell-CHSH local state (as well as absolutely three-settings unsteerable state) having a LHV model as well as LHS model under single and double interaction of the depolarizing channel. Note that since, $p_{2}<p_{1}$, the state $\rho_{i}(q, s)$ (with $q=0.34, s=0.97$ ) can be transformed into an absolutely Bell-CHSH local state (as well as an absolutely three-settings unsteerable state) having an LHV model as well as LHS model under double interaction of the depolarizing channel with smaller channel strength compared to that under single interaction.

\section{Steerability breaking channels and depolarizing map}

In [55], Bhattacharya et al. gave a sufficient criterion for unsteerability pertaining to a state in two qubits. They considered the following state in two qubits

$$
\chi=\frac{1}{4}\left(\mathbb{I} \otimes \mathbb{I}+\vec{a} . \vec{\sigma} \otimes \mathbb{I}+\sum_{i} t_{i i} \sigma_{i} \otimes \sigma_{i}\right)
$$

where $\mathbb{I}$ is the identity operator acting on $\mathbb{C}^{2}, \sigma_{i}$ s are the three Pauli matrices, $i \in\{1,2,3\}, \vec{r}, \vec{a}$ is vector in $\mathbb{R}^{3}$ with norm less than or equal to unity, $\vec{a} . \vec{\sigma}=\sum_{i=1}^{3} a_{i} \sigma_{i}$ and $t_{i i}=\operatorname{Tr}\left[\chi\left(\sigma_{i} \otimes \sigma_{i}\right)\right]$. The state in Eq. (11) is unsteerable if $\max _{\hat{x}}\left[(\vec{a} \cdot \hat{x})^{2}+2\|T \hat{x}\|\right] \leq 1$, where $\|\cdot\|$ is the Euclidean vector norm, $T=\left[t_{i j}\right]$ and $\hat{x}$ is a unit vector.

Since $\max _{\hat{x}}(\vec{a} \cdot \hat{x})^{2}=|\vec{a}|^{2}$ and $\max _{\hat{x}}\|T \hat{x}\|=\sqrt{\lambda_{\max }}$, where $\lambda_{\text {max }}$ is the largest eigenvalue of $T^{\dagger} T$, we obtain the condition as

$$
|\vec{a}|^{2}+2 \sqrt{\lambda_{\max }} \leq 1 .
$$

The depolarizing map is an important completely positive map in quantum information processing. It has been studied in the context of incompatibility breaking channels [40]. Its action on a single qubit system $A$ is given by

$$
\Gamma_{\epsilon}(A)=\epsilon A+(1-\epsilon) \frac{1}{2} \operatorname{Tr}[A] \mathbb{I}
$$


where $\epsilon$ is the channel parameter. Now, consider the state given in Eq. (11). Then due to the action of the depolarizing map on the state we obtain,

$$
\left(\Gamma_{\epsilon} \otimes \mathbb{I}\right) \chi=\frac{1}{4}\left(\mathbb{I} \otimes \mathbb{I}+\epsilon(\vec{a} . \vec{\sigma})+\sum_{i} \epsilon t_{i i} \sigma_{i} \otimes \sigma_{i}\right) .
$$

On using the unsteerability criterion, the resultant state is unsteerable if

$$
|\epsilon \vec{a}|^{2}+2 \epsilon \sqrt{\max \left(t_{11}^{2}, t_{22}^{2}, t_{33}^{2}\right)} \leq 1 .
$$

This provides a bound on the parameter $\epsilon$ for which the map becomes steerability breaking. One may also consider the map to be incompatibility breaking [40], as steerability and incompatibility of measurements have a one to one correspondence [41]. However, one must note that since the unsteerability criterion is only sufficient, the bounds are not tight.

\section{Conclusions}

We have discussed the possible detrimental effects of the environment on nonlocal resources because in the context of any quantum information protocol one cannot ignore environmental influences. We have documented various scenarios where nonlocality is lost, sometimes to the extent that it cannot be retrieved even with the strong resource of global unitary operations. We have illustrated various channel actions which destroy certain correlations. From the perspective of experiments, one should avoid those maps to preserve various nonlocal correlations. It is in this line of thought, that our work assumes significance. Precisely we show that there are instances where a quantum state loses its ability to violate the Bell-CHSH inequality or the three-settings steering inequality. This contributes to the notion of nonlocality breaking channels and also focusses on steerability breaking maps.

We also attempted to further the understanding of absolutely local maps, which render the resultant state useless regarding nonlocality. This is akin to the work already done in entanglement theory where absolute separability maps were considered [48]. In the tables presented, as expected intuitively, we observe that in most of the cases the parameter ranges obtained for a single interaction are subsets of that obtained for double interaction. We would like to reiterate that our work concerns mixed entangled states as pure states always violate some suitably chosen Bell's inequality.

We have laid down a theoretical proposal to generate entangled states admitting LHS model. As such entangled states occupy an intriguing existence in the theory of nonlocality and foundations of quantum theory, one might consider the experimental demonstration of the result. We have obtained bounds on the parameter of the depolarizing map for which it becomes steerability breaking (or one may also term them as incompatibility breaking). This does not depend on any particular inequality. This work also leads to certain open questions and possibilities for subsequent studies. For example, one may probe other channel parameters for which they become steerability breaking. An extension of the work in higher dimensions and multipartite systems also deserves attention.

\section{Acknowledgements}

We would like to gratefully acknowledge fruitful discussions with Professor Guruprasad Kar. Bihalan Bhattacharya acknowledges support from the DST-INSPIRE, Government of India. Debarshi Das acknowledges the financial support from University Grants Commission (UGC), Government of India. Nirman Ganguly would like to acknowledge support from the Research Initiation Grant of BITS-Pilani, Hyderabad vide letter no. BITS/GAU/RIG/2019/H0680 dated April 22, 2019. Archan S. Majumdar acknowledges Project no. DST/ICPS/Qust/2018/98 of Department of Science and Technology, Government of India.

\section{References}

[1] N. Brunner, D. Cavalcanti, S. Pironio, V. Scarani, S. Wehner. Bell nonlocality. Reviews of Modern Physics 2014; 86(2):419-478. doi:10.1103/ RevModPhys. 86.419.

[2] J. S. Bell. On the Einstein-Podolsky-Rosen paradox. Physics 1964; 1(3):195-200. CERN: 111654. doi: 10.1103/PhysicsPhysiqueFizika.1.195.

[3] J. F. Clauser, M. A. Horne, A. Shimony, R. A. Holt. Proposed experiment to test local hidden-variable theories. Physical Review Letters 1969; 23(15):880884. doi:10.1103/PhysRevLett.23.880.

[4] A. Fine. Hidden variables, joint probability, and the Bell inequalities. Physical Review Letters 1982; 48(5):291-295. doi : 10.1103/PhysRevLett. 48 , 291.

[5] A. Einstein, B. Podolsky, N. Rosen. Can quantummechanical description of physical reality be considered complete?. Physical Review 1935; 47(10):777780. doi:10.1103/PhysRev.47.777. 
[6] E. Schrödinger. Discussion of probability relations between separated systems. Mathematical Proceedings of the Cambridge Philosophical Society 1935; 31(4):555-563. doi:10.1017/ S0305004100013554.

[7] E. Schrödinger. Probability relations between separated systems. Mathematical Proceedings of the Cambridge Philosophical Society 1936; 32(3):446452. doi:10.1017/S0305004100019137.

[8] H. M. Wiseman, S. J. Jones, A. C. Doherty. Steering, entanglement, nonlocality, and the Einstein-Podolsky-Rosen paradox. Physical Review Letters 2007; 98(14):140402. arXiv:quant-ph/0612147. doi:10.1103/PhysRevLett.98.140402.

[9] S. J. Jones, H. M. Wiseman, A. C. Doherty. Entanglement, Einstein-Podolsky-Rosen correlations, Bell nonlocality, and steering. Physical Review A 2007; 76(5):052116. doi:10.1103/PhysRevA. 76.052116 .

[10] A. Milne, S. Jevtic, D. Jennings, H. Wiseman, T. Rudolph. Quantum steering ellipsoids, extremal physical states and monogamy. New Journal of Physics 2014; 16(8):083017. doi : 10.1088/1367$2630 / 16 / 8 / 083017$

[11] S. Jevtic, M. Pusey, D. Jennings, T. Rudolph. Quantum steering ellipsoids. Physical Review Letters 2014; 113(2):020402. doi:10.1103/ PhysRevLett.113.020402.

[12] S. Jevtic, M. J. W. Hall, M. R. Anderson, M. Zwierz, H. M. Wiseman. Einstein-Podolsky-Rosen steering and the steering ellipsoid. Journal of the Optical Society of America B 2015; 32(4):A40-A49. doi: $10.1364 /$ josab.32.000a40

[13] J. Bowles, T. Vértesi, M. T. Quintino, N. Brunner. One-way Einstein-Podolsky-Rosen steering. Physical Review Letters 2014; 112(20):200402. doi: 10.1103/PhysRevLett. 112.200402

[14] B. Wittmann, S. Ramelow, F. Steinlechner, N. K. Langford, N. Brunner, H. M. Wiseman, R. Ursin, A. Zeilinger. Loophole-free Einstein-PodolskyRosen experiment via quantum steering. New Journal of Physics 2012; 14(5):053030. doi : 10.1088/ 1367-2630/14/5/053030.

[15] D. A. Evans, H. M. Wiseman. Optimal measurements for tests of Einstein-Podolsky-Rosen steering with no detection loophole using twoqubit Werner states. Physical Review A 2014; 90(1):012114. doi:10.1103/PhysRevA.90. 012114

[16] P. Chowdhury, T. Pramanik, A. S. Majumdar, G. S. Agarwal. Einstein-Podolsky-Rosen steering using quantum correlations in non-Gaussian entangled states. Physical Review A 2014; 89(1):012104. doi: 10.1103/PhysRevA.89.012104.

[17] T. Pramanik, M. Kaplan, A. S. Majumdar. Finegrained Einstein-Podolsky-Rosen-steering inequalities. Physical Review A 2014; 90(5):050305. doi: 10.1103/PhysRevA.90.050305.

[18] P. Chowdhury, T. Pramanik, A. S. Majumdar. Stronger steerability criterion for more uncertain continuous-variable systems. Physical Review A 2015; 92(4):042317. doi:10.1103/PhysRevA. 92.042317 .

[19] D. Das, S. Datta, C. Jebaratnam, A. S. Majumdar. Cost of Einstein-Podolsky-Rosen steering in the context of extremal boxes. Physical Review A 2018; 97(2):022110. doi: 10.1103/PhysRevA. 97.022110.

[20] S. Sasmal, D. Das, S. Mal, A. S. Majumdar. Steering a single system sequentially by multiple observers. Physical Review A 2018; 98(1):012305. doi:10. 1103/PhysRevA.98.012305.

[21] M. D. Reid. Demonstration of the EinsteinPodolsky-Rosen paradox using nondegenerate parametric amplification. Physical Review A 1989; 40(2):913-923. doi:10.1103/PhysRevA.40. 913.

[22] Z. Y. Ou, S. F. Pereira, H. J. Kimble, K. C. Peng. Realization of the Einstein-Podolsky-Rosen paradox for continuous variables. Physical Review Letters 1992; 68(25):3663-3666. doi:10.1103/ PhysRevLett.68.3663.

[23] E. G. Cavalcanti, S. J. Jones, H. M. Wiseman, M. D. Reid. Experimental criteria for steering and the Einstein-Podolsky-Rosen paradox. Physical Review A 2009; 80(3):032112. doi:10.1103/ PhysRevA.80.032112.

[24] R. Horodecki, P. Horodecki, M. Horodecki, K. Horodecki. Quantum entanglement. Reviews of Modern Physics 2009; 81(2):865-942. doi: 10.1103/RevModPhys.81.865. 
[25] R. F. Werner. Quantum states with Einstein-Podolsky-Rosen correlations admitting a hidden-variable model. Physical Review A 1989; 40(8):4277-4281. doi:10.1103/PhysRevA.40.4277.

[26] M. T. Quintino, T. Vértesi, D. Cavalcanti, R. Augusiak, M. Demianowicz, A. Acín, N. Brunner. Inequivalence of entanglement, steering, and Bell nonlocality for general measurements. Physical Review A 2015; 92(3):032107. doi: 10.1103/PhysRevA. 92.032107 .

[27] C. H. Bennett, G. Brassard, C. Crépeau, R. Jozsa, A. Peres, W. K. Wootters. Teleporting an unknown quantum state via dual classical and EinsteinPodolsky-Rosen channels. Physical Review Letters 1993; 70(13):1895-1899. doi:10.1103/ PhysRevLett.70.1895.

[28] S. Pironio, A. Acín, S. Massar, A. B. de la Giroday, D. N. Matsukevich, P. Maunz, S. Olmschenk, D. Hayes, L. Luo, T. A. Manning, C. Monroe. Random numbers certified by Bell's theorem. $\mathrm{Na}$ ture 2010; 464(7291):1021-1024. doi: 10.1038/ nature09008.

[29] R. Colbeck, R. Renner. Free randomness can be amplified. Nature Physics 2012; 8(6):450-453. doi : $10.1038 /$ nphys 2300 .

[30] A. Chaturvedi, M. Banik. Measurement-deviceindependent randomness from local entangled states. Europhysics Letters 2015; 112(3). doi: 10.1209/ 0295-5075/112/30003.

[31] C. H. Bennett, G. Brassard. Quantum cryptography: public key distribution and coin tossing. Theoretical Computer Science 2014; 560(1):7-11. doi: $10.1016 /$ j.tcs.2014.05.025

[32] A. Shenoy-Hejamadi, A. Pathak, S. Radhakrishna. Quantum cryptography: key distribution and beyond. Quanta 2017; 6(1):1-47. doi:10.12743/ quanta.v6i1.57

[33] S. Popescu. Bell's inequalities and density matrices: revealing "hidden" nonlocality. Physical Review Letters 1995; 74(14):2619-2622. doi:10. 1103/PhysRevLett.74.2619.

[34] N. Gisin. Hidden quantum nonlocality revealed by local filters. Physics Letters A 1996; 210(3):151156. doi : 10 . 1016/S0375-9601(96)80001-6.
[35] T. Pramanik, Y.-W. Cho, S.-W. Han, S.-Y. Lee, Y.-S. Kim, S. Moon. Revealing hidden quantum steerability using local filtering operations. Physical Review A 2019; 99(3):030101. doi: 10.1103/PhysRevA. 99.030101 .

[36] P. W. Shor. Additivity of the classical capacity of entanglement-breaking quantum channels. Journal of Mathematical Physics 2002; 43(9):4334-4340. doi:10.1063/1.1498000

[37] M. Horodecki, P. W. Shor, M. B. Ruskai. Entanglement breaking channels. Reviews in Mathematical Physics 2003; 15(6):629-641. doi:10.1142/ s0129055x03001709.

[38] R. Pal, S. Ghosh. Non-locality breaking qubit channels: the case for CHSH inequality. Journal of Physics A: Mathematical and Theoretical 2015; 48(15):155302. doi:10.1088/1751-8113/48/ $15 / 155302$.

[39] Z.-W. Liu, X. Hu, S. Lloyd. Resource destroying maps. Physical Review Letters 2017; 118(6):060502. doi:10.1103/PhysRevLett.118.060502.

[40] T. Heinosaari, J. Kiukas, D. Reitzner, J. Schultz. Incompatibility breaking quantum channels. Journal of Physics A: Mathematical and Theoretical 2015; 48(43):435301. doi: 10. 1088/1751-8113/ $48 / 43 / 435301$

[41] R. Uola, C. Budroni, O. Gühne, J.-P. Pellonpää. Oneto-one mapping between steering and joint measurability problems. Physical Review Letters 2015; 115(23):230402. doi:10.1103/PhysRevLett. 115.230402 .

[42] K. Życzkowski, P. Horodecki, A. Sanpera, M. Lewenstein. Volume of the set of separable states. Physical Review A 1998; 58(2):883-892. doi:10.1103/PhysRevA.58.883.

[43] F. Verstraete, K. Audenaert, B. De Moor. Maximally entangled mixed states of two qubits. Physical Review A 2001; 64(1):012316. doi : 10.1103/ PhysRevA.64.012316.

[44] N. Johnston. Separability from spectrum for qubitqudit states. Physical Review A 2013; 88(6):062330. doi : 10.1103/PhysRevA.88.062330

[45] N. Ganguly, J. Chatterjee, A. S. Majumdar. Witness of mixed separable states useful for entanglement creation. Physical Review A 2014; 89(5):052304. doi:10.1103/PhysRevA.89.052304 
[46] A. Roy, S. S. Bhattacharya, A. Mukherjee, N. Ganguly. Characterization of nonlocal resources under global unitary action. Quantum Studies: Mathematics and Foundations 2018; 5(2):315-323. doi : 10.1007/s40509-017-0122-1.

[47] N. Ganguly, A. Mukherjee, A. Roy, S. S. Bhattacharya, B. Paul, K. Mukherjee. Bell-CHSH violation under global unitary operations: necessary and sufficient conditions. International Journal of Quantum Information 2018; 16(4):1850040. doi : 10.1142/s0219749918500405.

[48] S. N. Filippov, K. Y. Magadov, M. A. Jivulescu. Absolutely separating quantum maps and channels. New Journal of Physics 2017; 19(8):083010. doi: 10.1088/1367-2630/aa7e06.

[49] K. Mukherjee, B. Paul, D. Sarkar, A. Mukherjee, S. S. Bhattacharya, A. Roy, N. Ganguly. Generation of nonlocality 2017; arXiv: 1702.07782.

[50] S. S. Bhattacharya, A. Mukherjee, A. Roy, B. Paul, K. Mukherjee, I. Chakrabarty, C. Jebaratnam, N. Ganguly. Absolute non-violation of a threesetting steering inequality by two-qubit states. Quantum Information Processing 2017; 17(1):3. doi: 10.1007/s11128-017-1734-4.
[51] D. Cavalcanti, L. Guerini, R. Rabelo, P. Skrzypczyk. General method for constructing local hidden variable models for entangled quantum states. Physical Review Letters 2016; 117(19):190401. doi: 10.1103/PhysRevLett.117.190401.

[52] F. Hirsch, M. T. Quintino, T. Vértesi, M. F. Pusey, N. Brunner. Algorithmic construction of local hidden variable models for entangled quantum states. Physical Review Letters 2016; 117(19):190402. doi:10.1103/PhysRevLett.117.190402.

[53] R. Horodecki, P. Horodecki, M. Horodecki. Violating Bell inequality by mixed spin- $\frac{1}{2}$ states: necessary and sufficient condition. Physics Letters A 1995; 200(5):340-344. doi:10.1016/03759601(95)00214-n.

[54] M. A. Nielsen, I. L. Chuang. Quantum Computation and Quantum Information. 10th Edition. Cambridge University Press, Cambridge, 2010.

[55] J. Bowles, F. Hirsch, M. T. Quintino, N. Brunner. Sufficient criterion for guaranteeing that a two-qubit state is unsteerable. Physical Review A 2016; 93(2):022121. doi:10.1103/PhysRevA. 93.022121. 